\title{
Análisis de habilidades demostrativas que evidencian estudiantes de matemáticas de la UNAH
}

David Fernando Méndez Oyuela ${ }^{1}$

\section{RESUMEN}

En este estudio se pretende identificar y analizar habilidades que debe tener un estudiante para poder desarrollar la habilidad de realizar demostraciones matemáticas en el nivel de pregrado. Para ello, se realizó un estudio con estudiantes de la asignatura de Estructuras Algebraicas I del programa en Matemáticas en el nivel de Licenciatura de la Universidad Nacional Autónoma de Honduras. Con esto se pretende identificar dichas aptitudes, analizar si es posible potenciarlas y de qué manera se puede realizar tal tarea.

Palabras clave: demostración, competencia, álgebra, matemática educativa.

\section{ABSTRACT}

This study aims to identify and analyze skills that a student must have in order to perform mathematical demonstrations at the undergraduate level. To this end, a study was conducted with students of the first abstract algebra course of the Mathematics program at the Bachelor's level of the National Autonomous University of Honduras. This aims to identify these skills, analyze if it is possible to enhance them and in what way such a task can be carried out.

Keywords: demonstration, competence, algebra, mathematics education.

${ }^{1}$ Profesor del Departmento de Matematicas, Facultad de Ciencias, UNAH: david.mendez@unah.edu.hn 


\section{INTRODUCCIÓN}

La demostración en matemáticas juega un papel fundamental para darle rigor y formalidad a las teorías en las que se fundamentan muchas aplicaciones a la vida real, de tales aplicaciones dependen importantes procesos de la actividad humana, como los cálculos que se realizan en la ingeniería, los análisis financieros que hacen las empresas o el modelaje que se realiza en la economía; la ciencia en general se vale de dichas teorías para generar conocimiento, por lo que es de vital importancia que la teoría en matemática esté bien fundamentada.

En los cursos de matemáticas en el nivel superior, el nivel de formalidad que se presenta a los estudiantes en general se mantiene bajo, principalmente por las dificultades que representa para los estudiantes comprender y realizar demostraciones matemáticas.

En este estudio se pretende identificar y analizar las habilidades que debe tener un estudiante para poder realizar demostraciones matemáticas en el nivel de pregrado. Para ello, se realizará un estudio con estudiantes del programa en Matemáticas en el nivel de Licenciatura de la Universidad Nacional Autónoma de Honduras. Con esto se pretende identificar dichas habilidades, analizar si es posible potenciarlas y de qué manera se puede realizar tal tarea.

En términos generales una demostración matemática es una prueba a una conjetura o proposición, mediante una serie de razonamientos lógicos, partiendo de un conjunto de definiciones 0 axiomas ${ }^{2}$. Cualquier persona que ha intentado realizar una demostración matemática, habrá notado diferencia entre el tipo de habilidades que se necesitan para hacer demostraciones y para hacer, por ejemplo, ejercicios de aritmética, por lo que el tipo de análisis a realizar debe ser desde una perspectiva diferente.

En un trabajo realizado por Godino y Recio (1997) , propone: “...utilizaremos el término de 'prueba' para referirnos de modo genérico al objeto emergente del sistema de prácticas argumentativas (o argumentos) aceptadas en el seno de una comunidad, 0 por una persona, ante situaciones de validación y decisión, esto es, situaciones que requieren justificar el carácter de verdadero de un enunciado, o la eficacia de una acción" (pág. 2).

\footnotetext{
${ }^{2} \operatorname{Ver}($ Crespo, 2005), donde hace un resumen completo sobre las definiciones que brindan diferentes autores del concepto de demostración.
} 
En este estudio se utilizará esta postura con respecto al concepto de prueba, es muy importante destacar un hecho subjetivo que es intrínseco a la demostración: "es válida en el seno de una comunidad", es decir, su validez dependerá del entorno en el que se analice.

Duval (2002) afirma que: "probablemente, hacer demostraciones sea el reto más importante en el currículo de matemáticas, es un umbral que pocos estudiantes logran sobrepasar", párrafo que claramente muestra la magnitud del problema y la preocupación que genera en la comunidad de estudiosos de la Matemática Educativa.

En programas especializados de matemáticas (en Honduras, las licenciaturas en Matemáticas de la UNAH, la maestría en Matemáticas de la UNAH, el profesorado en matemáticas en el grado de licenciatura de la UPNFM y la maestría en Matemática Educativa de la UPNFM), se plantea al estudiante problemas que requieren hacer demostraciones. Pero las mismas no son consideradas una prioridad en los contenidos de asignaturas de Matemáticas de educación media, por lo que es común que se encuentren deficiencias en los estudiantes una vez que ingresan en el nivel superior.

En el Currículo Nacional Básico, documento que estructura los programas de Educación Media en el país, el estudio de la demostración se limita a una breve introducción a la demostración formal en geometría euclídea básica, utilizando el modelo de Van Hiele, método que se ha investigado que genera problemas de aprendizaje cuando los estudiantes se enfrentan a problemas relacionados con demostraciones (Usiskin, 1982).

Se observa que surge la necesidad de identificar y analizar, qué habilidades son necesarias para poder aprender a realizar demostraciones en matemáticas, en este estudio en particular, se trabajará el área del Álgebra Abstracta, por su carácter intrínseco demostrativo y con estudiantes de la carrera de Matemáticas de la Universidad Autónoma de Honduras. Éste análisis proveerá mejoras a la comprensión del problema, de manera que se puedan generar sugerencias sobre la enseñanza del concepto de la demostración.

Específicamente, este estudio busca responder a la siguiente interrogante:

¿Qué habilidades necesita un estudiante para que pueda desarrollar la habilidad de demostrar en Álgebra Abstracta?

Otra justificación que se puede resaltar es que después de la revisión de bibliografía, 
se han encontrado pocos o ningún estudio que específicamente analice las competencias demostrativas de estudiantes de pregrado en el ámbito del álgebra abstracta, de modo que esto además brindará un aporte a la matemática educativa.

\section{Conceptos teóricos}

De trabajos realizados por Godino (2002), se obtiene una definición simple del concepto de competencia matemática: "capacidad para realizar adecuadamente tareas matemáticas específicas", de modo que se adecúa dicha definición a nuestro contexto de la demostración. En este estudio se entenderá como competencia matemática demostrativa a la capacidad para realizar adecuadamente demostraciones matemáticas.

Se han realizado algunos estudios relacionados con clasificaciones de tipos de demostraciones, por ejemplo los trabajos realizados por Bell (1976), Balacheff (1988) y Harel y Sowder (2007), donde se identificó que las demostraciones se pueden clasificar como:

- Relacionadas con convencimiento externo

- Demostraciones empíricas

- Demostraciones deductivas

En este estudio, en base a esta clasificación estarán determinados los criterios para diferenciar la forma en que se hacen las demostraciones.

Referente a las estrategias que un estudiante utiliza para enfrentarse al nuevo reto de demostrar resultados generales, Alcock y Simpson (2002) definen las siguientes:

- Generalización.

- Abstracción de propiedades.

- Trabajar con las definiciones.

Muchos estudios, entre ellos los realizados por Martin y Harel (1989), Hersh (1993) y Hanna $(1995,1996)$ también han discutido sobre la diferencia entre una demostración formal e informal. En el proceso de enseñanza-aprendizaje de la demostración en Álgebra Abstracta se ha encontrado con estudiantes que fallan en distinguir entre una argumentación formal y una informal, de modo que percibimos una deficiencia en la definición de demostración en los estudiantes, cuestión que en nuestro ámbito pudiera ser generada por la poca experiencia que cuentan los estudiantes en el tema. 
Es de convención generalizada en el ámbito de las matemáticas que la lógica es la herramienta en el que los matemáticos se basan para construir sus demostraciones formales. La lógica brinda estructura a las demostraciones en matemáticas y provee de leyes para poder escribir proposiciones en formas equivalentes, de modo que una proposición en matemática se puede reescribir de formas distintas utilizando la inferencia lógica. La lógica tiene sus orígenes en los tiempos de Aristóteles y fue una herramienta de validación de argumentos, principalmente filosóficos. El párrafo "la lógica matemática es lógica tratada por métodos matemáticos, pero además, utilizamos la lógica en la matemática" de Kleene (1967), resume la relación simétrica que se da entre la lógica y la matemática, similar a la relación que se da entre, por ejemplo, la matemática y la física.

Es deseable que un estudiante que se vaya a enfrentar con demostraciones como las de álgebra abstracta tenga conocimiento de la lógica matemática y su relación con la argumentación que se hace en matemáticas, en el caso de este estudio los estudiantes cumplen con dicho requerimiento en cierta medida, en el sentido que el programa que cursan incluye una asignatura de Matemáticas Discretas, en la que usualmente se introduce al estudiante al mundo de la Lógica Matemática.

La falta de una metodología sistemática para la enseñanza y aprendizaje de la demostración es una de las principales situaciones que generan obstáculos para que los estudiantes logren aprehender el concepto de demostración. Es común que en los cursos donde se comienza a trabajar demostraciones formales en programas de Matemáticas, los docentes se limitan a realizar demostraciones en un pizarrón 0 presentarle a los estudiantes un conjunto de pruebas ya realizadas, con la esperanza de que los estudiantes puedan realizar un proceso de observación-imitación y que generen su propio aprendizaje. Este modelo favorece más que todo a los estudiantes que son talentosos por naturaleza, responde al paradigma tradicional de la enseñanza y obedece a la concepción platónica de la matemática (Godino, Batanero y Vincenc, 2002).

Finalizando este apartado, autores como Schoenfeld (1994) han resaltado que es importante añadir en el currículo de secundaria, estudios sobre demostraciones que hagan que los estudiantes comprendan la idea de que estas pueden ser vistas como una forma de pensar asociada a cualquier objeto matemático; señala que es deseable que un estudiante de secundaria sea capaz de sentirse cómodo haciendo demostraciones y de determinar cuándo un cierto argumento en realidad es una demostración. 


\section{Metodología de la investigación}

El enfoque de este trabajo es cualitativo y se puede describir como un estudio exploratorio, en el que se busca comprender experiencias de estudiantes de los primeros cursos de programas de pregrado en matemáticas, sobre la competencia demostrativa.

La población estudiada son los estudiantes de cursos de matemáticas en los que se trabajan problemas relacionados con demostraciones, específicamente en el área de álgebra abstracta.

El estudio se desarrolló en la Universidad Nacional Autónoma de Honduras (UNAH), se trabajará con estudiantes pasantes del curso de Estructuras Algebraicas I, parte del plan de estudios del programa de Matemáticas en el grado de licenciatura de la UNAH, durante jornadas de trabajo con duración variable (de una hasta tres horas). El motivo de escoger esta muestra reside en que este es el primer curso donde los estudiantes se enfrentan con demostraciones matemáticas formales en el programa de estudios mencionado. Además resultaba sumamente viable puesto que es el lugar de trabajo del autor del trabajo.

La principal técnica de recolección de información es la observación de actividades que se desarrollaron con el grupo de estudiantes. La justificación principal de esto es que este tipo de actividades son las que usualmente confronta el individuo de interés. Específicamente, se realizó una prueba diagnóstica y tres actividades de recolección de información.

Cuando se le presentó a los estudiantes con las actividades, que consistieron en problemas de realizar demostraciones de diferentes tipos y con objetivos específicos, en primera instancia se buscó identificar si los estudiantes se encasillan en alguna de las estrategias presentadas por Alcock y Simpson (2002) y discutidas anteriormente en los aspectos teóricos. La segunda fase del análisis consistió en determinar las dificultades y obstáculos que los estudiantes presentaron. Por último se determinará si los estudiantes cuentan o no con las habilidades necesarias para el desarrollo de las demostraciones, contrastando su desempeño con el modelo de lo que se espera que se desarrolle en cada actividad. 
Figura 1. Fases del análisis

\section{Elección de estrategia}

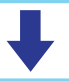

Búsqueda de obstáculos

\section{Análisis de las habilidades demostrativas}

La siguiente matriz muestras los indicadores específicos correspondientes a las categorías de análisis mencionadas anteriormente. Además se incluyen la fuente de información y los instrumentos utilizados.

\section{Tabla 1. Matriz de categorías}

\begin{tabular}{|l|l|l|l|}
\hline Categoría & Indicadores & $\begin{array}{l}\text { Fuentes de } \\
\text { información }\end{array}$ & Instrumentos \\
\hline $\begin{array}{l}\text { 1. Elección de } \\
\text { estrategia }\end{array}$ & $\begin{array}{l}\text { Identifica alguna estrategia } \\
\text { para realizar la } \\
\text { demostración. }\end{array}$ & $\begin{array}{l}\text { Aplicación de prueba } \\
\text { diagnóstica. }\end{array}$ & $\begin{array}{l}\text { Prueba } \\
\text { diagnóstica. }\end{array}$ \\
\hline $\begin{array}{l}\text { 2. Búsqueda de } \\
\text { obstáculos }\end{array}$ & $\begin{array}{l}\text { a) No puede dar un ejemplo } \\
\text { de una situación en la que } \\
\text { se cumpla el enunciado. }\end{array}$ & $\begin{array}{l}\text { Actividades de } \\
\text { b) Crea contraejemplos } \\
\text { para expresar que una } \\
\text { proposición es falsa. }\end{array}$ & \\
\hline & $\begin{array}{l}\text { c) Considera que un caso } \\
\text { particular es una prueba de } \\
\text { un enunciado general. }\end{array}$ & & \\
\hline $\begin{array}{l}\text { 3. Análisis de la } \\
\text { competencia } \\
\text { demostrativa }\end{array}$ & $\begin{array}{l}\text { En caso que realiza una } \\
\text { supuesta prueba, evidencia } \\
\text { que maneja secuencias } \\
\text { lógicas de argumentos. }\end{array}$ & $\begin{array}{l}\text { Tres actividades de } \\
\text { trabajo con diferentes } \\
\text { tipos de problemáticas } \\
\text { relacionadas a } \\
\text { demostraciones. }\end{array}$ & \\
\hline
\end{tabular}

\section{Análisis de resultados}

En forma resumida, de la prueba diagnóstica se determinó que los estudiantes de la muestra mostraron en forma vaga una asociación de las estrategias que se utilizan para hacer demostraciones matemáticas para realizar otro tipo de problemas, por ejemplo, toma ejemplos de situaciones particulares para luego generalizar, transfor- 
ma el problema en un problema más pequeño y que luego le permita mostrar el resultado global. Además, los estudiantes evidencian que tienen una visión formal del concepto de demostración, es decir, no se convencen con afirmaciones vagas sobre un resultado, buscan utilizar razonamientos lógicos que le permitan validar sus afirmaciones.

La primera actividad consistió de una prueba en la que se le presenta al estudiante la demostración del Algoritmo de Euclides que aparece en el libro de Herstein (1988), se le pide al estudiante que lea cuidadosamente la demostración y que la trate de entender, para que luego responda a 7 preguntas que están planteadas con el objetivo de recabar información sobre si los estudiantes identifican las componentes descritas anteriormente. El algoritmo de Euclides es una propiedad de los números enteros que es común que se introduzca en los libros para cursos de Álgebra Ábstracta a nivel de pregrado, como preparación preliminar para estudiar la teoría de grupos.

La actividad se le realizó a 23 estudiantes de la asignatura de Estructuras Algebraicas I de la Universidad Nacional Autónoma de Honduras. Los estudiantes participaron de forma voluntaria durante una hora, el docente del curso afirmó que el Algoritmo de Euclides ya se había estudiado en la clase. La segunda pregunta que se realizó en la actividad fué: ¿Le parece evidente lo que afirma el teorema? ¿Considera necesaria la demostración? ¿Por qué?

Esta pregunta tiene como objetivo determinar si los estudiantes comprenden el enunciado y además obtener información sobre si los estudiantes tienen necesidad de ver una prueba para convencerse del resultado, como afirma Lutzer (2005), hay estudiantes que se conforman con realizar un poco de experimentación y la experiencia es suficiente para aceptar algunas afirmaciones.

De los 23 estudiantes, 12 consideraban evidente lo que presentaba el problema y de ellos todos consideraban necesaria la demostración, es decir, los estudiantes están convencidos de que a pesar que una afirmación sea evidente, se necesita demostrarla para lograr formalidad.

En una de las preguntas un estudiante confiesa que probablemente el problema sean los conocimientos previos que tiene sobre los enteros, afirma (copiado textualmente):

"si lo que me cuesta ver cuando disen $m-(q+1) n$ está en $V$, a pesar que $m-$ $(\mathrm{q}+1) \mathrm{n}<\mathrm{r} \Rightarrow \Leftarrow$, me imagino que no lo entiendo por falta de bases que no tengo". 
Cabe destacar en este sentido, que el curso de Estructuras Algebraicas I incluye una introducción a los números enteros, teoría de conjuntos y funciones, para luego comenzar con la teoría de grupos. Antes de esta clase, el plan de estudios no incluye un curso donde se estudien a profundidad los conjuntos de números, razón que podría justificar en cierta medida lo que dice este estudiante.

La quinta pregunta que se realizó fué: Brinde un ejemplo que ilustre el teorema y otro de un caso donde no se cumpla. Con esta pregunta se asume que si los estudiantes comprenden un teorema, podrán brindar ejemplos de cuando el mismo se cumple y casos donde si se viola alguno de los requisitos, la conclusión puede fallar, es decir, que un estudiante comprenda que el resultado general, incluye todos los casos particulares.

Para poder brindar lo requerido, los estudiantes tienen que ser capaces de comprender el enunciado, es decir, de determinar cuál es la hipótesis y cuál es la tesis de la proposición. En este caso, la mayoría de estudiantes logró brindar un ejemplo de cuando se cumple el teorema, relacionándolo incluso con el procedimiento de división de enteros que se enseña en la escuela.

Por otro lado, la mayoría de estudiantes no pudo dar un ejemplo de cuando el teorema no se cumple, esto quiere decir que los estudiantes no logran identificar la hipótesis de la proposición, es decir la parte: " $m$ y $n$ son enteros y $n>0$ ", de aquí con solo tomar $\mathrm{m}$ y $\mathrm{n}$ no enteros, se podría obtener lo requerido.

Concluímos que los estudiantes no logran comprender bien la estructura de un enunciado, porque no pudieron identificar casos en los que no se cumpliera la hipótesis del mismo.

También se preguntó a los estudiantes: ¿Qué estrategia considera que se usó en la demostración? Se concluyó que los estudiantes en general si manejan un panorama básico sobre las estrategias lógicas que se usan para hacer demostraciones.

En la segunda actividad se optó por presentar a los individuos una prueba incompleta, con espacios por rellenar, con el objetivo principal de determinar si los estudiantes pueden seguir la estructura típica que tiene una demostración. La demostración trata de probar que un conjunto de aplicaciones lineales es un grupo.

De los 19 estudiantes, 10 estaban convencidos de que llenar los espacios resulta en una prueba a la proposición. Es decir, estos 10 estudiantes no consideran necesarios 
más detalles, se convencen de que, por ejemplo, como ya se brinda el elemento inverso, ese debe ser, no necesitan comprobarlo detalladamente. Sin embargo, algunos estudiantes si necesitan convencerse de lo mismo, por ejemplo, en la actividad se afirmaba: "Finalmente, el elemento inverso es $(T a, b)-1=$ Ta $-1,-a-1 b$ ", es decir, ya se brindaba la forma de calcular el elemento inverso, pero una estudiante realizó lo siguiente (copiado textualmente):

$" \Longrightarrow(\mathrm{Ta}, \mathrm{b})-1(\mathrm{Ta}, \mathrm{b})=\mathrm{T} 10 \quad=\mathrm{Ta}-1,-\mathrm{a}-1 \mathrm{~b} * \mathrm{Ta}, \mathrm{b}=\mathrm{T} 10 \quad=-$ $\mathrm{Ta}-1 \mathrm{a}, \mathrm{a}-1 \mathrm{~b}-\mathrm{a}-1 \mathrm{~b}=\mathrm{T} 1,0 \bigotimes "$

como se observa, necesitó verificar (a su manera) si lo que se mencionaba era verdadero, es decir, la estudiante utiliza un enfoque empírico de la demostración, no se conforma con que se le mencione un hecho, no ve la demostración desde el punto de vista de convencimiento externo, la autoridad no es suficiente (Harel y Sowder, 2007).

Una de las preguntas en este caso fué ¿Puede dar una descripción gráfica del conjunto? ¿Qué efecto tendría la operación? esta interrogante busca determinar si los estudiantes logran visualizar un efecto gráfico en la aplicación de la operación. En este sentido, la pregunta tiene un corte exploratorio, es decir, se esperaría que algunos estudiantes realizaran algunos dibujos de rectas (asumiendo que logran identificarlas como rectas) y que busquen darle algún sentido a la operación (que en la prueba se especifica que es la operación de composición).

De los 19 estudiantes, 12 realizaron algún dibujo representando rectas en el plano, otros estudiantes consideraron que el conjunto era todo $\mathbb{R} 2$ puesto que el conjunto de todas las rectas lo recubren.

Posiblemente la respuesta más acertada la brindó el siguiente estudiante:

Figura 2. Pendiente e intercepto

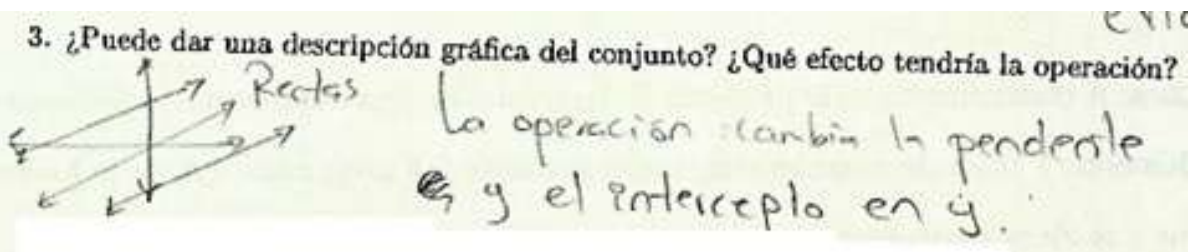


Esta respuesta no brinda pistas sobre si este estudiante le da un sentido gráfico a la operación, pero definitivamente es una descripción generalizada de lo que la composición de rectas en el plano hace. Se concluye sobre esta pregunta que, como menciona Duval (2002), lograr un vínculo entre representaciones gráficas y la comprensión de un enunciado es un juego duro, lo estudiantes encuentran difícil relacionar ambos objetos abstractos.

Otra pregunta hecha fué la siguiente: ¿Es G abeliano? Si cree que no, ¿puede dar un contraejemplo? de los 19 estudiantes, 7 brindaron un contraejemplo, 5 lo demostraron de forma general y el resto no logró ninguna de los dos casos. Es decir, un buen número de estudiantes tiene la habilidad demostrativa de brindar contraejemplos. Además de poder dar contraejemplos, algunos de los estudiantes si asociaban el hecho con que eso es suficiente para comprobar que la respuesta es negativa, es decir, construían correctamente contraejemplos, así, la habilidad "brindar contraejemplos" se vuelve más amplia, se transforma en "brindar contraejemplos para demostrar la falsedad de una proposición". Un estudiante brindó el siguiente contraejemplo:

$\mathrm{T} 1,2=\mathrm{x}+2$

$\mathrm{T} 4,8=4 \mathrm{x}+8$

$\mathrm{T} 1,2(\mathrm{~T} 4,8)(\mathrm{x})=(4 \mathrm{x}+8)+2=4 \mathrm{x}+10$

$\mathrm{T} 4,8(\mathrm{~T} 1,2)(\mathrm{x})=(4(\mathrm{x}+2))+8=4 \mathrm{x}+16$

La actividad final consistió de el siguiente problema:

PROBLEMA: Sea $S$ un conjunto infinito $y$ $G=\{f: S \rightarrow S, f$ es $1-1$ y $f(s) \neq s$ para un número finito de elementos $s \in S\}$ Demuestre que $\mathrm{G}$ es un grupo respecto a la operación $\circ$, la composición de funciones.

La actividad se aplicó durante una hora a un grupo de 30 estudiantes del curso de Estructuras Algebraicas I anteriormente descrito, a los estudiantes ya se les había presentado los conceptos que se requieren para resolver el problema: grupos, subgrupos y funciones. Los resultados fueron contundentes, de los 30 estudiantes, ninguno pudo completar la demostración, sin embargo, algunos de ellos si completaron algunos de los detalles que se requieren. Se esperaba que los estudiantes aplicaran las definiciones de grupo y funciones inyectivas para ver que el conjunto requerido cumple con todos los requisitos para ser un grupo. Otra estrategia que hubiese sido válida era considerar el grupo $\mathrm{H}=(\operatorname{Perm}(\mathrm{S})$, o) y demostrar que $\mathrm{G}$ es subgrupo de $H$, donde Perm(S) $=\{\mathrm{f}: \mathrm{S} \rightarrow \mathrm{S} / \mathrm{f}$ es $1-1\}$, este grupo ya se había 
presentado a los estudiantes anteriormente y además conocían que para comprobar que un subconjunto es subgrupo, solo se deben de comprobar dos de los cuatro axiomas de grupo, a saber, la cerradura y la existencia de inversos.

Sin embargo, ninguno de los estudiantes optó por esta estrategia, los 30 estudiantes intentaron demostrar los cuatro axiomas de grupo para G. Es importante destacar que incluso 3 estudiantes concluyeron que el conjunto no era un grupo, cuestión que en un problema del tipo planteado pasa poco, puesto que la proposición está en el sentido de realizar la comprobación de una afirmación, no sobre la verdad o falsedad de la misma. Un estudiante escribió (copiado textualmente):

"Una contradicción. Al querer encontrar el elemento Neutro de G, El Elemento Neutro, posee una característica, que no es compatible con la propiedad que cumplen los elementos de G",

este estudiante evidencia que tiene problemas para analizar la condición $\mathrm{f}(\mathrm{s}) \neq \mathrm{s}$ para un número finito de elementos de $S$, puesto que si iS es la función de identidad de $S$ tenemos que i(s) = s para todo $s \in S$ y así iS $\in$ G porque iS $(s) \neq s$ para ningún elemento de $\mathrm{S}$, así el número finito de tales elementos es 0 .

De forma similar pero siendo más explícito, otro estudiante de los que consideraron que $\mathrm{G}$ no era grupo escribió (copiado textualmente):

"Elemento Neutro. $\mathrm{f}(\mathrm{h}(\mathrm{s}))=\mathrm{f}(\mathrm{s})=(\mathrm{f} \circ \mathrm{h})(\mathrm{s}), \mathrm{h}(\mathrm{s})=\mathrm{s} \Rightarrow$ El elemento neutro es "S" pero debido a la condición $\mathrm{f}(\mathrm{s}) \neq \mathrm{s}$ para un num. finito de elementos $\mathrm{s} \in \mathrm{S}$ concluyo en que el elemento "S" no siempre existira",

este estudiante confunde cuáles son los elementos del grupo, puesto que afirma el elemento neutro es " $S$ ", de este modo, está considerando que $S$ es un elemento de $\mathrm{G}$, cuestión que es imposible. A este respecto se puede afirmar que este estudiante está trabajando la demostración usando las definiciones, pero no tiene bien claros los conceptos y esto hace que falle en su demostración (Alcock y Simpson, 2002).

Otro punto importante es que muchos de los estudiantes, cuando comprobaban la cerradura, consideraban suficiente con decir que la composición de funciones es inyectiva(hecho que se les demostró en clase), pero olvidaban la condición clave: "f(s) $\neq \mathrm{s}$ para un número finito de elementos $\mathrm{s} \in \mathrm{S}$ ", por ejemplo, un estudiante hace el siguiente análisis: 
Figura 3. Una condición

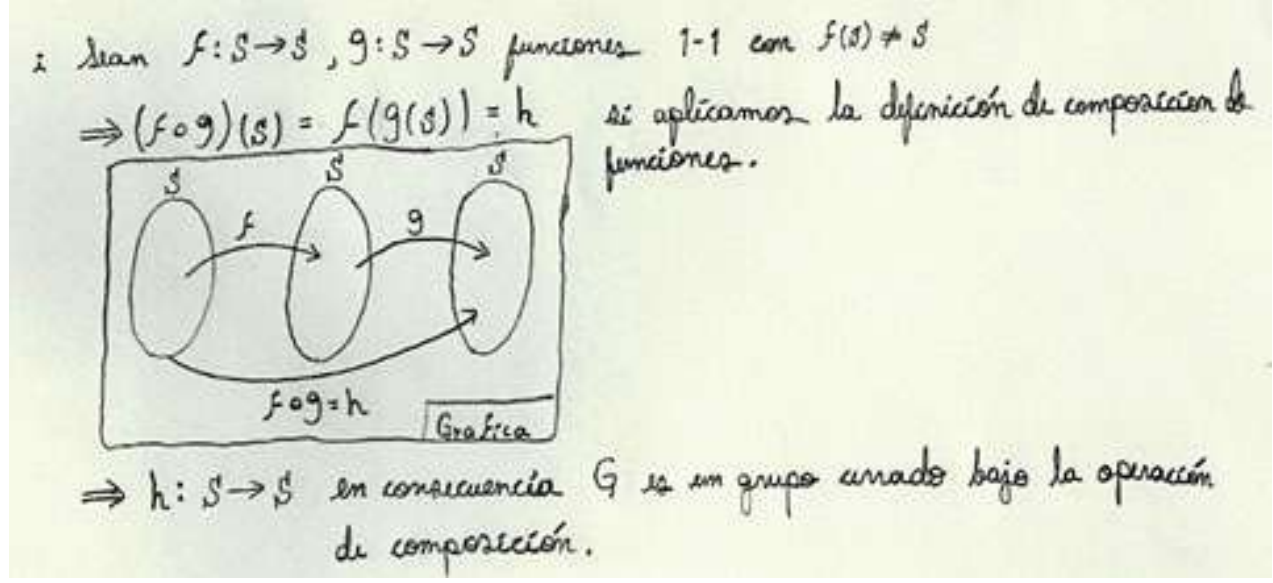

Este estudiante evidencia comprender bien el concepto de composición de funciones pero no chequea que la misma cumple con la condición mencionada anteriormente y así la prueba está incompleta. Se puede afirmar que este estudiante y todos los que no consideraron la condición, no lograron entender el enunciado y de ahí es complicado esperar que pudiesen realizar una buena demostración, como afirma Godino (2002b), la competencia matemática debe complementarse con la comprensión matemática de las técnicas necesarias para realizar las tareas.

Una de las preguntas fue: Si ha tenido dificultades para realizar la prueba, ¿cuáles han sido?, ¿Cómo cree que se podrían evitar? de los 30 estudiantes, 3 no respondieron a la pregunta y el resto respondieron que si tuvieron dificultades. Cabe destacar que 12 de los estudiantes respondieron que sus dificultades se deben a falta de preparación, porque afirmaron que la forma en que podrían solventar las dificultades que tuvieron es estudiar o practicar más los contenidos, por ejemplo un estudiante escribió (copiado textualmente):

"Falta de conocimiento de conceptos necesarios para su resolución. Mejoraría con mas estudio",

de modo que estos estudiantes solo reafirman algo esperado: no se puede esperar que exista una competencia matemática si no hay práctica y preparación. 
Figura 4. Estadística

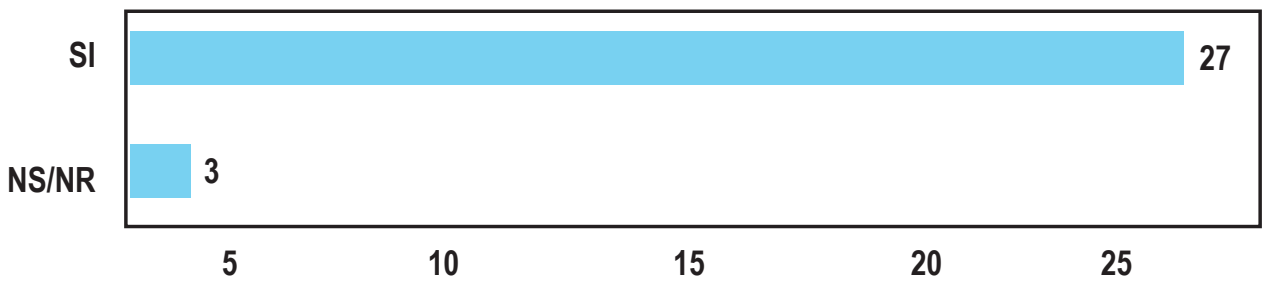

Por otro lado, otros estudiantes fueron más específicos respecto a las dificultades que tuvieron, por ejemplo, dos estudiantes afirmaron que tuvieron problemas con la parte que menciona sobre el conjunto infinito, otros dos mencionaron que la notación les generó problemas y cinco estudiantes afirmaron que su dificultad fué para ver cuáles eran los elementos identidad e inverso. A este respecto se puede mencionar que, según la literatura consultada, es una dificultad para los estudiantes la transición a los problemas demostrativos, además, ya dentro del ámbito demostrativo, también se dan transiciones que generan dificultades, por ejemplo, pasar de probar una estructura "pruebe que x es X" a otra estructura "pruebe que x satisface la definición de X" (Alcock y Simpson, 2002).

Así, estos estudiantes, según su trabajo en la actividad y según las justificaciones que dan para mostrar dificultades, demuestran estar pasando una transición similar, donde no solo se hacen pruebas simples en las que un objeto $x$ satisface la propiedad $\mathrm{X}$, si no, donde se le pide comprobar que un objeto más abstracto que $\mathrm{x}$ (en este caso, el conjunto $\mathrm{G}$ ) satisface una definición con varias exigencias $X$ (las propiedades para ser grupo).

En definitiva, en esta actividad se logró captar que algunas habilidades necesarias para realizar demostraciones no necesariamente se van a adquirir por pura repetición de un ejercicio o por imitación de técnicas realizadas por los profesores o autores de libros, se observa una necesidad de presentar al estudiante de forma explícita esta situación, aunque no sea necesariamente en un curso dedicado exclusivamente a ello, los profesores durante los cursos podrían realizar esta tarea.

Finalmente, la última pregunta fue la siguiente: ¿Qué importancia considera usted que tiene en su carrera el poder realizar demostraciones como esta? el objetivo de esta pregunta es indagar sobre el significado que tiene la demostración para los estudiantes que participaron y además, la motivación que tienen para aprender a realizarlas. Cabe notar primeramente que todos los estudiantes que contestaron consideran que la demostración es una herramienta muy importante para su carrera 
y que es necesario que obtengan la competencia para realizarlas. Se analizan algunas de las respuestas que llaman la atención, por ejemplo, un estudiante escribió (copiado textualmente):

"Es importante porque devemos saber de donde se hace cada cosa, no solo irnos a como nos enseñaban en la escuela",

este estudiante muestra que en su pensamiento existe una diferencia entre el tipo de enseñanza que recibe en la escuela al tipo de enseñanza en el nivel superior, comprende que en el nivel primario y secundario de educación la enseñanza que recibió fué sin hacer hincapié en demostrar los resultados que se aprendían y que más bien solo hay que aceptarlos. En este sentido, se puede resaltar una contradicción que se da en la matemática, por un lado, se puede considerar a las matemáticas como el área más racional de todas, puesto que sus conclusiones se validan por la razón, pero por otro lado el razonamiento detrás de la matemática a veces no es comprendido por el estricto rigor y simbolismo abstracto que se necesita para la formalidad, en este caso se vuelve el área más irracional y autoritaria. De aquí que la brecha entre la matemática del nivel primario y medio y la del nivel superior es tan abrupta (Duval, 2002).

Otra conclusión que se puede generar sobre las respuestas a esta interrogante es que a pesar de que los estudiantes consideran que la demostración es la herramienta fundamental a manejar, no están seguros de cómo hacerlo y no muestran un interés legítimo por desarrollar técnicas para hacer demostraciones, más bien se interesan más por el contenido de los teoremas, cuestión que no es negativa ni mucho menos, pero que genera una forma asistemática de desarrollar la competencia demostrativa.

\section{CONCLUSIONES}

Del análisis de las actividades de recolección de información se obtienen las siguientes conclusiones generales:

1. Se observó que las principales habilidades para realizar demostraciones en álgebra abstracta en el nivel de pregrado son:|begin_inset Separator latexparlend_inset

1. Entender el enunciado.

2. Abstraer ideas. 
3. Realizar conclusiones generales sobre las particulares.

4. Brindar ejemplos y contraejemplos.

5. Manejo de estrategias diferentes para realizar demostraciones.

6. Visualización de objetos abstractos (cuando es factible).

7. Asociar enunciados con proposiciones lógicas.

2. Los estudiantes perciben a la demostración como la herramienta principal a aprender a manejar durante el desarrollo de su carrera, esto es llamativo considerando que algunos de los estudiantes del curso optan por una orientación en Estadística, rama que en el campo laboral usualmente no usa mucho de la demostración como herramienta(en el sentido abstracto de usarla para comprobar proposiciones 0 teoremas).

3. Los estudiantes consideran que en situaciones la demostración es la herramienta de aprendizaje y no el objeto, esto se debe al enfoque que se utiliza en el programa que estudian, esto por supuesto es positivo, pero se podría aprovechar más con una mejor enseñanza de la demostración.

4. Los estudiantes con los que se trabajó expresan que una de las dificultades principales para desarrollar habilidades demostrativas es que consideran que no tienen las herramientas suficientes para lograr un aprendizaje significativo de las mismas, ya que no hay una formación especializada en el tema.

5. En cierta medida, los estudiantes manejan las estructuras básicas que se usan para hacer demostraciones, como las pruebas usando definiciones y resultados, las pruebas por inducción o las pruebas por reducción al absurdo, pero esto no es suficiente para realizar demostraciones más complejas en las que se hacen combinaciones de las técnicas o cuando hay que construir objetos abstractos para lograr un objetivo.

6. Se considera que la principal habilidad previa para hacer demostraciones en álgebra abstracta es la del manejo de conceptos requeridos para estudiar el área, por ejemplo, se identifican los conceptos relacionados con los números enteros, la teoría de conjuntos o el concepto y propiedades de las funciones.

7. Se necesitan más datos y un estudio diacrónico para una validación más fuerte de estas conclusiones y del estudio en general.

\section{REFERENCIAS BIBLIOGRÁFICAS}

Alcock L. y Simpson A. (2002). Definitions: Dealing with Categories Mathematically. For the learning of mathematics, p. 28-34.

Balacheff N. (1988). Aspects of proofs in pupils' practice of school mathematics. Mathematics, 
teachers and children, p. 216-235.

Bell A. (1976). A study of pupils' proof-explanations in mathematical situations. Educational Studies in Mathematics, p. 23-40.

Crespo C. (2005). El papel de las argumentaciones matemáticas en el discurso escolar. La estrategia de deducción por reducción al absurdo. (Tesis de Maestría). CINVESTAV, México.

Duval, R. (2002). Proof understanding in mathematics: What ways for students?. Proceedings of 2002 International Conference on Mathematics: Understanding Proving and Proving to Understand, p. 61-67.

Godino, J. (2002a). Competencia y comprensión matemática: ¿qué son y cómo se consiguen?. Revista de didáctica de las matemáticas, p. 9-19.

Godino, J. (2002b). Perspectiva semiótica de la competencia y comprensión matemática. La matematica e la sua didattica, p. 434-450.

Godino, J. y Recio, A. (1997). Meaning of proofs in mathematics education. Proceedings of the 21th International Conference of PME, p. 313-321.

Godino, J., Batanero, C. y Vincenc, F. (2002). Fundamentos de la enseñanza y el aprendizaje de las matemáticas para maestros. Granada, España: ReproDigital.

Hanna, G. (1995). Challenges to the importance of proof. For the Learning of Mathematics, p. 42-49.

Harel, G. y Sowder, L. (2007). Toward comprehensive perspectives on the learning and teaching of proof. Second Handbook of Research on Mathematics Teaching and Learning, p. 805-842.

Hersh, R. (1993). Proving is convincing and explaining. Educational Studies in Mathematics, p. 389-399.

Herstein, I. (1988). Álgebra Abstracta. México, D.F., México: Grupo Editorial Iberoamérica.

Lutzer, C. (2005). The role of proof in mathematics and mathematics education. Problems, Resources and Issues in Mathematics undergraduate Studies, p. 374.

Martin, G. y Harel, G. (1989). Proof frames of preservice elementary teachers. Journals of Research in Mathematics Education, p. 41-51.

Schoenfeld, A.(1994). What do we know about mathematics curricula? . Journal of Mathematical Behavior, p. 55-80.

Usiskin, Z. (1982). Van Hiele Levels and Achievement in Secondary School Geometry. Chicago, USA: University of Chicago. 